\title{
Counting 1D free electron laser growing modes in the presence of space charge
}

\author{
G. Wang, ${ }^{1, *}$ V. N. Litvinenko, ${ }^{1,2}$ and S. Webb ${ }^{3}$ \\ ${ }^{1}$ Collider-Accelerator Department, Brookhaven National Laboratory, Upton, New York 11973-5000, USA \\ ${ }^{2}$ Department of Physics \& Astronomy, Stony Brook University, Stony Brook, New York 11794-3800, USA \\ ${ }^{3}$ Tech-X Corporation, 5621 Arapahoe Avenue Suite A, Boulder, Colorado 80303, USA
}

(Received 14 September 2012; published 17 December 2012)

\begin{abstract}
Knowing the number and structure of the free electron laser's (FEL) growing modes is of fundamental importance to its theoretical description and its applications. The one-dimensional FEL dispersion relation, including the space-charge effects, is well established in the FEL theory, but questions remain about the number of the growing solutions (modes). In this paper, we provide the definite answers to the latter question and identify the amplification bandwidth of these modes.
\end{abstract}

DOI: 10.1103/PhysRevSTAB.15.120701

PACS numbers: 41.60.Cr

\section{INTRODUCTION}

Free electron lasers (FELs) are known to amplify coherent radiation near its resonant wavelength of

$$
\lambda_{0} \cong \frac{\lambda_{w}}{2 \gamma^{2}}\left(1+a_{w}^{2}\right) \equiv \frac{\lambda_{w}}{2 \gamma_{z}^{2}},
$$

where $\lambda_{w}$ is the FEL's wiggler period, $a_{w}$ is the dimensionless wiggler parameter, and $\gamma=E_{0} / m c^{2}$ is the electron beam's relativistic factor.

By assuming the electron beam has a uniform density distribution, electrons move along identical trajectories, and the amplified wave is a monochromatic plane wave, the FEL process can be described by a set of self-consistent linear 1D FEL equations, i.e., the set of Maxwell equations and the linearized Vlasov equation. ${ }^{1}$ The most general method of solving these equations was developed by Saldin et al. [2]. These authors also introduced a set of normalized variables, many of which we use in this paper. Specifically, all lengths are normalized as $\hat{z}=\Gamma z$, using the 1D FEL gain parameter ${ }^{2}$

\footnotetext{
*gawang@bnl.gov
}

Published by the American Physical Society under the terms of the Creative Commons Attribution 3.0 License. Further distribution of this work must maintain attribution to the author(s) and the published article's title, journal citation, and DOI.

The applicability region of the 1D FEL model is $\varepsilon_{\perp}<$ $\lambda /(4 \pi), L_{G}<L_{R}=\pi w_{0}^{2} / \lambda$, and $L_{e}>N_{u} \lambda$, where $\varepsilon_{\perp}$ is the transverse electron beam emittance, $\lambda$ is the radiation wavelength, $L_{G}=\Gamma^{-1}$ is the 1D gain length, $w_{0}$ is the electron beam radius, and $L_{e}$ is the electron beam bunch length [1]. In addition, linearization of the Vlasov equation requires that the perturbation of the electrons' phase space density distribution due to the FEL process is small compared with that of the unperturbed distribution.

${ }^{2}$ For a planar wiggler, the JJ term [2] should be included, which does not change the FEL's dispersion relation, i.e. the focus of this paper.

$$
\Gamma=\left[\frac{\pi j_{0} a_{w}^{2} \omega}{c \gamma_{z}^{2} \gamma^{3} I_{A}}\right]^{1 / 3}
$$

where $c$ is the speed of light, $j_{0}$ is the electron beam current density, $I_{A}=m_{e} c^{3} / e$ is the Alfvén current, and $\omega=$ $2 \pi c / \lambda$ is the FEL's radiation frequency. The space-charge effects are described by the dimensionless plasma-wave parameter

$$
\hat{\Lambda}_{p} \equiv \frac{1}{\Gamma}\left[\frac{4 \pi j_{0}}{\gamma_{z}^{2} \gamma I_{A}}\right]^{1 / 2}
$$

while the dimensionless detuning from the FEL resonance is described by ${ }^{3}$

$$
\hat{\Delta} \equiv-\frac{1}{\Gamma}\left[k_{w}+\frac{\omega}{c}-\frac{\omega}{v_{z}}\right],
$$

where $k_{w}=2 \pi / \lambda_{w}$ and $v_{z}=c \sqrt{1-\gamma_{z}^{-2}}$ is the effective longitudinal velocity of electrons in the FEL wiggler.

The method developed in [2] consists of reducing the set of equations to a single linear integrodifferential equation, and then applying the Laplace transformation:

$$
f(s)=\int_{0}^{\infty} f(\hat{z}) e^{-s \hat{z}} d \hat{z},
$$

where $\hat{z}$ is the normalized coordinate along the FEL's axis. This technique reduces the solution of the self-consistent 1D FEL equations to finding the solutions of the dispersion relation $(\mathrm{DR})$ :

$$
s=\frac{D(s, \hat{\Delta})}{1-i \hat{\Lambda}_{p}^{2} D(s, \hat{\Delta})},
$$

where

\footnotetext{
${ }^{3}$ Here we change the sign of notation used in [2] to $\hat{\Delta} \equiv-\hat{C}$ for high frequencies to correspond to positive detuning $\hat{\Delta}>0$.
} 


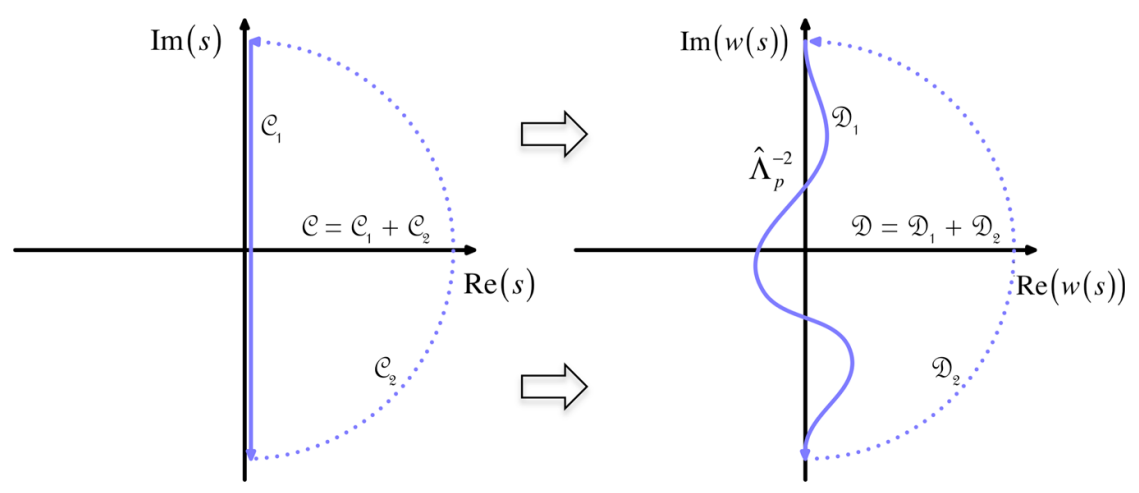

(a)

(b)

FIG. 1. Mapping from $s$ to $w(s)$ as defined in Eq. (7). The map from $\mathcal{C}_{2}$ to $\mathcal{D}_{2}$ approaches an identity map when $|s| \rightarrow \infty$.

$$
D(s, \hat{\Delta}) \equiv \int_{-\infty}^{\infty} d \hat{P} \frac{d \hat{F}(\hat{P})}{d \hat{P}} \frac{1}{s+i(\hat{P}-\hat{\Delta})} \quad \text { for } \operatorname{Re}(s)>0
$$

is defined by the electrons' energy-distribution function, $\hat{F}(\hat{P})$, and the dimensionless energy deviation is defined as $\hat{P} \equiv\left(E / E_{0}-1\right) / \rho$, with $\rho=\gamma_{z}^{2} \Gamma c / \omega$ being the Pierce parameter. The resulting evolution of the electron density modulation as well as the radiation field in the FEL can be described as a direct sum of the modes

$$
f(z, \hat{\Delta})=\sum_{i} f_{i}(\hat{\Delta}) e^{s_{i}(\hat{\Delta}) \hat{z}},
$$

where $s_{i}$ are the solutions of the dispersion relation (4) and $f_{i}$ are defined by the initial conditions (see [2]). The growing solutions with $\operatorname{Re}\left(s_{i}\right)>0$ are of most interest for the FELs since they dominate the nongrowing solutions in a high-gain FEL [3].

Saldin and colleagues [2] derived the dispersion relation (4) for a 1D FEL with collinear propagation of the electron and FEL's plane wave of radiation. In our recent paper [4], we expanded this derivation to include the plane waves propagating at an angle with respect to the motion of the electron beam, i.e.,

$$
\vec{k}=k_{z} \vec{e}_{z}+\vec{k}_{\perp} .
$$

We showed that Eqs. (4) and (5) remain correct with our substitution of

$$
\hat{\Delta}_{3 \mathrm{D}} \equiv \hat{\Delta}+\hat{k}_{\perp}^{2}
$$

where $\overrightarrow{\hat{k}}_{\perp} \equiv \sqrt{\frac{\rho}{2}} \frac{\vec{k}_{\perp}}{\gamma_{z} \Gamma}$. Hence, solving the DR for a 1D FEL is equivalent to finding its solution for a noncollinear FEL with a spatially uniform electron beam.

In our recent paper [3], taking an approach of the Nyquist plot [5], we developed a method of determining the number of growing modes and calculating their high-frequency cutoff for 1D FEL in the absence of the space-charge effect. In this paper, we extend our approach to study in detail the 1D FEL's dispersion relations, including the space-charge effects. We derive constraints on the maximal number of growing modes and upper frequency cutoff for a FEL with a spatially uniform electron beam having an arbitrary energy distribution. ${ }^{4}$

\section{THE METHOD}

Any solution of Eq. (4) with $\operatorname{Re}(s)>0$ corresponds to a growing mode whose amplitude increases as $\sim \exp \left[\operatorname{Re}\left(s_{i}\right) \hat{z}\right]$, with $\operatorname{Re}\left(s_{i}\right)$ being the growth rate of mode $i$. For compactness, later in the text we drop $\hat{\Delta}$ from the DR expressions and its roots, while assuming their implicit dependence on $\hat{\Delta}$. To explore the roots of Eq. (4), we define a complex function,

$$
w(s) \equiv s-D(s)\left(1+i s \hat{\Lambda}_{p}^{2}\right)
$$

and consider a mapping of a contour $\mathcal{C}$, shown in Fig. 1(a), from the complex $s$ plane to the complex $w(s)$ plane. The contour $\mathcal{C}$ comprises a vertical straight line parallel to the imaginary axis, $\mathcal{C}_{1}$, and a semicircle in the right-half complex plane, $\mathcal{C}_{2}$. A contour $\mathcal{D}$ shown in Fig. 1(b) is one of the possible maps of $\mathcal{C}$, where $\mathcal{D}_{1}$ and $\mathcal{D}_{2}$, respectively, are the maps of $\mathcal{C}_{1}$ and $\mathcal{C}_{2}$. If $w(s)$ is a meromorphic function in the right-half plane of $\operatorname{Re}(s)>0$ with no poles or zeros along $\mathcal{C}$, the argument principle (the change in the argument of a complex function along a closed contour) states that the following expression gives the winding number:

$$
W \equiv \frac{1}{2 \pi} \oint d\{\arg [w(s)]\}=Z-P,
$$

where $Z$ is the number of roots and $P$ is the number of poles of $w(s)$, enclosed by the contour $\mathcal{C}$, counting multiplicity. As we discussed in our previous paper [3], the poles of $w(s)$

\footnotetext{
${ }^{4}$ See the constraints on energy distributions in the following section.
} 
are identical to those of $D(s)$ and located in the left-half plane of $\operatorname{Re}(s)<0 .^{5}$

We can prove directly, following the method indicated in [6], that for energy distributions satisfying

$$
\int_{-\infty}^{\infty}\left|\frac{d}{d \hat{P}} \hat{F}(\hat{P})\right| d \hat{P}<\infty
$$

since the derivative of $D(s)$ for $\operatorname{Re}(s)>0$,

$$
\begin{aligned}
\left|\frac{d}{d s} D(s)\right| & =\left|\int_{-\infty}^{\infty} d \hat{P} \frac{d \hat{F}(\hat{P})}{d \hat{P}} \frac{1}{[s+i(\hat{P}-\hat{\Delta})]^{2}}\right| \\
& \leq \frac{1}{\operatorname{Re}(s)^{2}} \int_{-\infty}^{\infty} d \hat{P}\left|\frac{d \hat{F}(\hat{P})}{d \hat{P}}\right|,
\end{aligned}
$$

is bounded, it ensures that $D(s)$ is an analytic function in the right-half plane. ${ }^{6}$ Hence, $w(s)$ does not have poles in the right-half plane and the winding number is equal to the number of growing roots ${ }^{7}$

$$
Z=W \text {. }
$$

\section{UPPER LIMIT ON THE NUMBER OF GROWING ROOTS}

According to Eq. (9), counting the number of growing roots of DR is reducible to finding the winding number of contour $\mathcal{D}$. Let us first discuss the properties of $\mathcal{D}_{2}$. We previously proved that for an energy distribution, $\hat{F}(\hat{P})$, satisfying

$$
\hat{F}(\hat{P}) \leq \frac{\hat{F}_{\max }}{1+\hat{P}^{2} / \hat{q}^{2}} \quad \text { for } \forall \hat{P} \in \mathbb{R},
$$

where $\hat{F}_{\max }$ and $\hat{q}$ are some positive numbers, the dispersion function (5) is diminished at infinity [3]:

$$
\lim _{|s| \rightarrow \infty}|\hat{D}(s)| \leq \sqrt{2} \pi \hat{q} \hat{F}_{\text {max }} \lim _{|s| \rightarrow \infty} \frac{1}{|s|^{2}}\left[1+\frac{\hat{q}}{\operatorname{Re}(s)}\right] .
$$

Hence, the difference between the map of Eq. (7) and an identity map $w(s)=s$ vanishes at $|s| \rightarrow \infty$ :

$$
\begin{aligned}
\lim _{|s| \rightarrow \infty}\left|\left(1+i s \hat{\Lambda}_{p}^{2}\right) D(s)\right| & \leq \lim _{|s| \rightarrow \infty}|D(s)|+\hat{\Lambda}_{p}^{2} \lim _{|s| \rightarrow \infty}|s||D(s)| \\
& =0
\end{aligned}
$$

i.e., the contour $\mathcal{D}_{2}$ is identical to $\mathcal{C}_{2}$. The contour $\mathcal{C}_{1}$ can be parametrized as

\footnotetext{
${ }^{5}$ As discussed in [6], this is a general property of susceptibility due to the causality.

${ }^{6}$ The analytical function is meromorphic by definition and hence, the argument principle is applicable.

${ }^{7}$ As the contour $\mathcal{C}$ stays in the right-half plane where $w(s)$ is analytic, there is no pole along $\mathcal{C}$. Assuming that all zeros of $w(s)$ at the imaginary axis of $s$ plane are isolated, there also are no zeros along $\mathcal{C}$. Further, if a root has multiplicity $N$, it corresponds to $N$ growing solutions, which generally are the product of a polynomial of order $N-1$, and a growing exponent.
}

$$
s=\varepsilon_{+}+i t
$$

with $\varepsilon_{+}$being an infinitesimal positive number and $t$ being a real number changing from $\infty$ to $-\infty$. Hence, taking the limit $\varepsilon_{+} \rightarrow 0$ at $\mathcal{C}_{1}$ yields ${ }^{8}$

$$
\begin{aligned}
D(i t)= & -i \int_{-\infty}^{\infty} d \hat{P} \frac{d \hat{F}(\hat{P})}{d \hat{P}} \frac{1}{t-\hat{\Delta}+\hat{P}} \\
= & \left.\pi \frac{d \hat{F}(\hat{P})}{d \hat{P}}\right|_{\hat{P}=\hat{\Delta}-t}-i P V \int_{-\infty}^{\infty} d \hat{P} \frac{d \hat{F}(\hat{P})}{d \hat{P}} \\
& \times \frac{1}{t-\hat{\Delta}+\hat{P}},
\end{aligned}
$$

and the following expression describes the map from $\mathcal{C}_{1}$ to $\mathcal{D}_{1}$ :

$$
\begin{aligned}
w(i t)= & i\left[t+\left(1-t \hat{\Lambda}_{p}^{2}\right) P V \int_{-\infty}^{\infty} d \hat{P} \frac{d \hat{F}(\hat{P})}{d \hat{P}} \frac{1}{t-\hat{\Delta}+\hat{P}}\right] \\
& -\left.\left(1-t \hat{\Lambda}_{p}^{2}\right) \pi \frac{d \hat{F}(\hat{P})}{d \hat{P}}\right|_{\hat{P}=\hat{\Delta}-t}
\end{aligned}
$$

As $t$ monotonically changes from $\infty$ to $-\infty, \mathcal{D}_{1}$ intersects with the imaginary axis when $\operatorname{Re}[w(i t)]=0$, i.e., at points where

$$
\left(1-t \hat{\Lambda}_{p}^{2}\right) \hat{F}^{\prime}(\hat{\Delta}-t)=0
$$

Following from the first multiplier in Eq. (15), one of the intersection points is independent of detuning and always occurs at a fixed point with

$$
t=t_{0}=\hat{\Lambda}_{p}^{-2},
$$

and

$$
w\left(i t_{0}\right)=i \hat{\Lambda}_{p}^{-2} .
$$

All other intersecting points are determined by the extrema of the energy-distribution function: ${ }^{9}$

$$
\left.\hat{F}^{\prime}\left(p_{n}\right) \equiv \frac{d \hat{F}(\hat{P})}{d \hat{P}}\right|_{p_{n}}=0, \quad n=1, \ldots, N,
$$

i.e., at

\footnotetext{
${ }^{8}$ If the energy-distribution function, $\hat{F}(\hat{P})$, is a smooth upperbounded function, the principal value integral always converges (Appendix A).

${ }^{9}$ Whether $\mathcal{D}_{1}$ crosses into the other half plane at the intersecting point is determined by the following expression:

$$
\left.\frac{d}{d t} \operatorname{Re}[w(i t)]\right|_{t=t_{n}}=\left.\hat{\Lambda}_{p}^{2}\left(p_{n}+\hat{\Lambda}_{p}^{-2}-\hat{\Delta}\right) \pi \frac{d^{2} \hat{F}(\hat{P})}{d \hat{P}^{2}}\right|_{\hat{P}=p_{n}} .
$$

As an extrema requires the second derivative to be nonzero, $\mathcal{D}_{1}$ always crosses into the other half plane at the intersecting points for $p_{n} \neq \hat{\Delta}-\hat{\Lambda}_{p}^{-2}$. In the case of $p_{n}=\hat{\Delta}-\hat{\Lambda}_{p}^{-2}$, the fixed intersecting point merges with the intersecting point at $t_{n}$, and since the above derivative vanishes, $\mathcal{D}_{1}$ stays in the same half plane after intersecting with the imaginary axis.
} 


$$
t=t_{n}=\hat{\Delta}-p_{n} .
$$

Hence, the number of extrema of the energy-distribution function determines the number of intersections. If $\hat{F}(\hat{P})$ is a smooth function satisfying $\hat{F}(\hat{P})_{\hat{P} \rightarrow \pm \infty} \rightarrow 0$ (i.e., a physically valid distribution), then $t_{n}$ always are discrete numbers. When the number of extrema, $N$, is finite, it is always odd since $\hat{F}(\hat{P})_{\hat{P} \rightarrow \pm \infty} \rightarrow 0$ requires the number of minima to be equal to that of maxima, $M$, minus one, i.e., ${ }^{10}$

$$
N=2 M-1 \text {. }
$$

Adding the fixed intersecting point of Eq. (16) makes the total number of the intersections of $\mathcal{D}_{1}$ with the imaginary axis even and equal to $2 M$. Since each clockwise or counterclockwise winding requires the contour to intersect twice with the imaginary axis, the maximal winding number cannot exceed half of the number of intersections. Thus, we proved the following theorem.

Theorem 1.-For a 1D FEL driven by an electron beam with energy distribution described by a smooth function, $\hat{F}(\hat{P}) \in C^{\infty}$, with $M$ maxima, if the energy-distribution function satisfies the following constraints:

$$
\int_{-\infty}^{\infty}\left|\frac{d}{d \hat{P}} \hat{F}(\hat{P})\right| d \hat{P}<\infty
$$

and

$$
\hat{F}(\hat{P}) \leq \frac{\hat{F}_{\max }}{1+\hat{P}^{2} / \hat{q}^{2}}, \quad \forall \hat{P} \in \mathbb{R}
$$

for some positive number $\hat{F}_{\max }$ and $\hat{q}$, the number of growing roots of its dispersion relation, $Z$, is no larger than $M$, i.e.,

$$
Z \leq M
$$

\section{COUNTING THE NUMBER OF GROWING ROOTS}

Equation (14) suggests that the location of the intersections, and therefore the winding number, depends upon detuning from the resonance. As we detail later, by investigating where $\mathcal{D}_{1}$ intersects with the imaginary axis, we can determine the frequency regions for FEL instability. To proceed, we enumerate the extrema of the distribution in the ascending order,

$$
p_{1}<p_{2}<\cdots<p_{n}<p_{n+1}<\cdots<p_{2 M-1},
$$

which makes $t_{n}$ a descending sequence. We note that the odd numbers of $n$ correspond to the maxima, and the even ones to the minima.

\footnotetext{
${ }^{10}$ While mathematically valid, distribution functions with infinite number of extrema, such as $a \frac{1+\cos (k \hat{P})}{1+\hat{P}^{2} / q^{2}}$, are only of academic interest and so are not considered further in this paper.
}

We emphasize that the values of the dispersion function (13) at the intersection points are constants, fully determined by the energy-distribution function:

$$
\begin{aligned}
& D\left[i\left(\hat{\Delta}-p_{n}\right), \hat{\Delta}\right]=-i P V \int_{-\infty}^{\infty} \frac{d \hat{F}(\hat{P})}{d \hat{P}} \frac{d \hat{P}}{\hat{P}-p_{n}} \equiv i D_{n}, \\
& \quad \text { for } n=1, \ldots, 2 M-1 .
\end{aligned}
$$

Hence, the values of $w\left(i t_{n}\right) \equiv i w_{n}$ at the intersection points are linear functions of the detuning $\hat{\Delta}$, with

$$
\begin{gathered}
w_{n}=A_{n} \hat{\Delta}-B_{n} \quad \text { for } n=1, \ldots, 2 M-1, \\
A_{n}=1+\hat{\Lambda}_{p}^{2} D_{n},
\end{gathered}
$$

and

$$
B_{n}=A_{n} p_{n}+D_{n}
$$

To determine the topology of $\mathcal{D}_{1}$, we start by investigating its locations at $t \rightarrow \pm \infty$. At $t \rightarrow+\infty, \operatorname{Im}[w(i t)] \rightarrow \infty$ and

$$
\begin{aligned}
& \operatorname{Re}[w(i t)]=\pi\left(\hat{\Lambda}_{p}^{2} t-1\right) \hat{F}^{\prime}(\hat{\Delta}-t)>0, \\
& \quad \text { for } t>\max \left(\hat{\Lambda}_{p}^{-2}, \hat{\Delta}-p_{1}\right)
\end{aligned}
$$

since $\hat{F}^{\prime}(\hat{P})>0$ for $\hat{P}<p_{1}$. Similarly, at $t \rightarrow-\infty$, $\operatorname{Im}[w(i t)] \rightarrow-\infty$, and

$$
\begin{aligned}
& \operatorname{Re}[w(i t)]=\pi\left(\hat{\Lambda}_{p}^{2} t-1\right) \hat{F}^{\prime}(\hat{\Delta}-t)>0, \\
& \quad \text { for } t<\min \left(\hat{\Lambda}_{p}^{-2}, \hat{\Delta}-p_{2 M-1}\right)
\end{aligned}
$$

since $\hat{F}^{\prime}(\hat{P})<0$, for $\hat{P}>p_{2 M-1}$. Thus, when $t$ changes from $+\infty$ to $-\infty, \mathcal{D}_{1}$ crosses from the upper-right-half plane into the left-half plane, intersects with the imaginary axis $2 M$ times $^{11}$ and returns to the lower-right-half plane. While the relative sequence of the $t_{n}$ remains unchanged with the detuning $\hat{\Delta}$, they move together with respect to $t_{0}$ as the detuning changes, and each $t_{n}$ passes $t_{0}$ at the detuning of

$$
\hat{\Delta}=\hat{\Delta}_{n} \equiv \hat{\Lambda}_{p}^{-2}+p_{n}
$$

with the point of the passing in the $w(s)$ plane given by

$$
w_{n}\left(\hat{\Delta}_{n}\right)=w_{0}=\hat{\Lambda}_{p}^{-2} .
$$

There are $2 M$ detuning intervals separated by $\hat{\Delta}_{n}$ and we assign an index $l(\hat{\Delta})$ to each of them with $l(\hat{\Delta})$ determined by

\footnotetext{
${ }^{11}$ That is except for a degenerated case with any intersecting point merging with the fixed intersecting point. Then, the contour $\mathcal{D}_{1}$ touches the imaginary axis without crossing it, reducing by one the maximal winding number, however, it does not change the overall sequence of intersections.
} 


$$
l(\hat{\Delta})=\sum_{n=1}^{2 M-1} \theta\left(\hat{\Delta}-\hat{\Lambda}_{p}^{-2}-p_{n}\right)
$$

where $\theta(x)$ is the Heaviside step function defined as

$$
\theta(x)= \begin{cases}0, & \text { for } x<0 \\ 1, & \text { for } x \geq 0\end{cases}
$$

For a series of intersections occurring at $t$ with a descending sequence of

$$
t_{1}, \ldots, t_{l}, t_{0}, t_{l+1}, \ldots, t_{2 M-1},
$$

the winding number is determined by the following expression (Appendix B):

$$
W(\hat{\Delta})=(-1)^{l(\hat{\Delta})}+\sum_{n=1}^{2 M-1}(-1)^{n+\theta[l(\hat{\Delta})-n]} \theta\left(A_{n} \hat{\Delta}-B_{n}\right) .
$$

For $\hat{\Delta} \rightarrow-\infty$ and for $A_{n} \neq 0$, the sequence of Eq. (34) is $\left\{t_{0}, t_{1}, \ldots, t_{2 M-1}\right\}$ and

$$
W_{-} \rightarrow 1+\sum_{n=1}^{2 M-1}(-1)^{n} \theta\left(-A_{n}\right)
$$

Similarly, for $\hat{\Delta} \rightarrow+\infty$ and $A_{n} \neq 0$, the sequence $\left\{t_{1}, \ldots, t_{2 M-1}, t_{0}\right\}$ yields

$$
W_{+} \rightarrow-1+\sum_{n=1}^{2 M-1}(-1)^{n+1} \theta\left(A_{n}\right) .
$$

Thus, for $A_{n} \neq 0$ the number of the growing modes at very large detuning is determined fully by the set of the signs of the $A_{n}$. In the case of all $A_{n}=1+\hat{\Lambda}_{p}^{2} \cdot D_{n}>0$, Eqs. (36) and (37) reduce to

$$
W_{-}=1 ; \quad W_{+}=0,
$$

signifying that the FEL amplification has a high-frequency cutoff.

According to Eq. (35), a change of the $W(\hat{\Delta})$ value seemingly could happen when $t_{0}$ advances within the sequence of Eq. (34). However, according to Eq. (32), when $t_{l}(\hat{\Delta})$ passing $t_{0}, \operatorname{Im}\left[w_{l}\left(\hat{\Delta}_{l}\right)\right]>0$ and the change in the value of $W(\hat{\Delta})$ at $\hat{\Delta}_{l}=\hat{\Lambda}_{p}^{-2}+p_{l}$ vanishes:

$$
\begin{aligned}
\delta W_{l} & =\left[W\left(\hat{\Delta}_{l}+\varepsilon\right)-W\left(\hat{\Delta}_{l}-\varepsilon\right)\right]_{\varepsilon \rightarrow o_{+}} \\
& =2(-1)^{l}\left\{1-\theta\left[\operatorname{Im} w_{l}\left(\hat{\Delta}_{l}\right)\right]\right\}=0 .
\end{aligned}
$$

Consequently, the changes of $W$ occur only when the imaginary part of $w\left(i t_{n}\right), A_{n} \cdot \hat{\Delta}-B_{n}$, changes sign at ${ }^{12}$

$$
\hat{\Delta}=\hat{\Delta}_{n}^{+} \equiv \frac{B_{n}}{A_{n}}=p_{n}+\frac{D_{n}}{1+\hat{\Lambda}_{p}^{2} D_{n}},
$$

\footnotetext{
${ }^{12}$ The case of $A_{n}=0$ is trivial, as there is no change of the sign and, naturally, no corresponding change occurs.
}

that entail a change in winding number by

$$
\begin{aligned}
\delta W_{n} & =\left\{W\left(\hat{\Delta}_{n}^{+}+\varepsilon\right)-W\left(\hat{\Delta}_{n}^{+}-\varepsilon\right)\right\}_{\varepsilon \rightarrow 0_{+}} \\
& =(-1)^{n+\theta(l-n)}\left[\theta\left(A_{n}\right)-\theta\left(-A_{n}\right)\right] .
\end{aligned}
$$

If all $\hat{\Delta}_{n}^{+}$differ, the winding number always jumps a step of one unit as the detuning varies. In the case of degeneration, i.e., when two or more $\hat{\Delta}_{n}^{+}$coincide, viz. $\hat{\Delta}_{n_{1}}^{+}=$ $\hat{\Delta}_{n_{2}}^{+}=\cdots=\hat{\Delta}_{n_{k}}^{+}$, the jump is a direct sum of the terms in Eq. (41):

$$
\delta W_{\mathrm{deg}}=\sum_{j=1}^{k}(-1)^{n_{j}+\theta\left(l-n_{j}\right)}\left[\theta\left(A_{n_{j}}\right)-\theta\left(-A_{n_{j}}\right)\right] .
$$

The number of such jumps, $K$, is less than or equal to the number of extrema, i.e.,

$$
K \leq 2 M-1 .
$$

Next, we define a new series, $d_{m}$ for $m=0,1,2, \ldots$, $K+1$, that includes all $\hat{\Delta}_{n}^{+}$in ascending order with $-\infty$ and $+\infty$ being, respectively, its first and its last element:

$$
d_{0}=-\infty<d_{1} \leq \cdots \leq d_{K}<d_{K+1}=\infty .
$$

There are $K+1$ detuning intervals separated by the elements of series $d_{m}$, and within each interval, the number of growing roots remains constant, i.e.,

$$
W_{m}=W(\hat{\Delta}) \quad \text { for } \hat{\Delta} \in\left[d_{m}, d_{m+1}\right) .
$$

It follows from the definition that $W_{0}=W_{-}$and $W_{K+1}=$ $W_{+}$, with $W_{ \pm}$defined in Eqs. (36) and (37) for $A_{n} \neq 0$.

From these analyses, for an arbitrary smooth distribution function having $M$ maxima, the following steps could be taken to determine the number of growing modes as a function of the detuning: (i) calculate the location of the $2 M-1$ extrema $p_{n}$; (ii) Evaluate the dispersion values, $D_{n}$, from Eq. (25) at these points; (iii) determine the $K+1$ set of intervals by calculating and sorting the values of $\hat{\Delta}_{n}^{+}$ using Eq. (40); (iv) assess the number of growing modes in each interval using Eq. (35).

Figure 2 shows the typical shape of the dependence of the number of growing modes on detuning for an FEL with

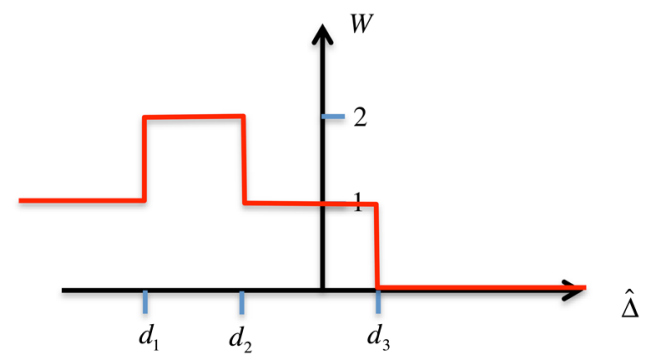

FIG. 2. A typical dependence of the number of growing modes on detuning for an FEL with an energy distribution having two maxima. 


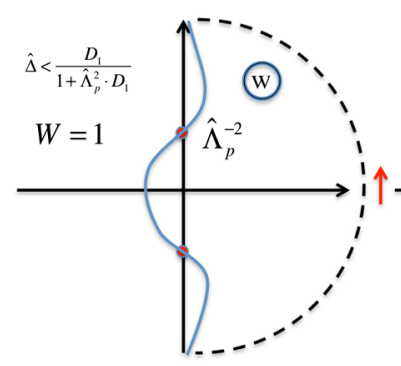

(a)

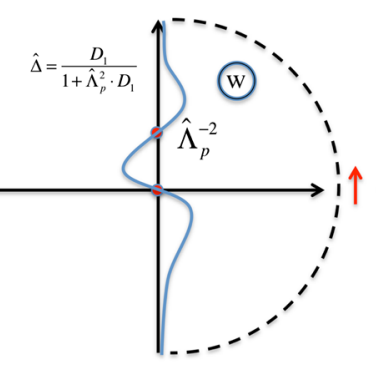

(b)

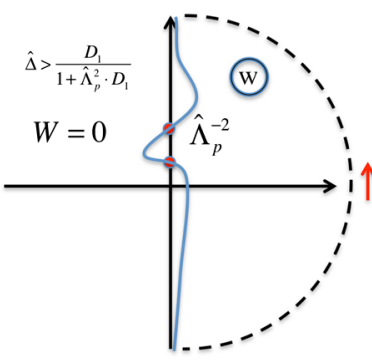

(c)

FIG. 3. Schematic drawing of possible $\mathcal{D}_{1}$ mapping when $\hat{F}(\hat{P})$ has only one extrema. (a) Low or negative detuning yields a winding number of 1, i.e., one growing mode. Part (b) corresponds to the cutoff frequency for FEL instability. (c) Above the threshold the winding number is 0 , i.e., there are no growing modes.

energy distribution with two maxima. It has four segments of detuning where $W$ remains constant. At large negative detuning, the FEL has a single growing mode; in a second interval it has two growing modes; and, in the third interval their number is reduced to one. Finally, in the fourth interval, at large positive detuning $\hat{\Delta}>d_{3}$, there is no growing mode. Thus, $d_{3}$ will determine the high-frequency cutoff for such a FEL.

In the following sections, we present some cases and examples, where the conclusions can go beyond those established in this section.

\section{ENERGY DISTRIBUTION WITH ONE MAXIMUM}

The theorem we developed in previous sections provides the strongest limitation for a FEL driven by electron beam with a bell-shaped energy distribution. ${ }^{13}$ There, the maximal number of growing modes is one and the highfrequency cutoff of FEL amplification can be obtained. As derived in Eqs. (16) and (19), one intersection occurs at $t_{0}=\hat{\Lambda}_{p}^{-2}$ with $w_{0}=\hat{\Lambda}_{p}^{-2}$ and the other at $t_{1}=\hat{\Delta}-p_{1}$ with

$$
w_{1}(\hat{\Delta})=\left(1+\hat{\Lambda}_{p}^{2} D_{1}\right) \hat{\Delta}-\left(1+\hat{\Lambda}_{p}^{2} D_{1}\right) p_{1}-D_{1} .
$$

Since $\hat{F}^{\prime}(\hat{P})>0$ for $\hat{P}<p_{1}$ and $\hat{F}^{\prime}(\hat{P})<0$ for $\hat{P}>p_{1}$, it follows that

$$
D_{1}=-P V \int_{-\infty}^{\infty} d \hat{P} \frac{d \hat{F}(\hat{P})}{d \hat{P}} \frac{1}{\hat{P}-p_{1}}>0
$$

and, hence, $w_{1}(\hat{\Delta})$ is a growing function of $\hat{\Delta}$. As $\hat{\Delta}$ increases, the sign of $w_{1}(\hat{\Delta})$ changes from negative to positive at

$$
\hat{\Delta}=\hat{\Delta}^{+} \equiv p_{1}+\frac{D_{1}}{1+\hat{\Lambda}_{p}^{2} D_{1}} .
$$

\footnotetext{
${ }^{13}$ We define the bell-shaped function as a smooth, positive, bounded function diminishing at infinity with a single maximum.
}

As $\hat{\Delta}^{+}$splits detuning into two regions with no changes of winding number in each region, we can insert $\hat{\Delta}= \pm \infty$ into Eq. (35) and obtain

$$
W= \begin{cases}1 & \text { for } \hat{\Delta}<\hat{\Delta}^{+} \\ 0 & \text { for } \hat{\Delta}>\hat{\Delta}^{+}\end{cases}
$$

The schemes drawn in Fig. 3 clarify the conclusion of Eq. (48). We note that for an energy distribution with one maximum,

$$
t_{1}>t_{0} \Leftrightarrow \operatorname{Im}\left[w\left(i t_{1}\right)\right]>\operatorname{Im}\left[w\left(i t_{0}\right)\right],
$$

and hence, as $t$ decreases, $\mathcal{D}_{1}$ always passes the upper intersection point first as illustrated in Fig. $3 .^{14}$

To summarize, we formulate the case of a 1D FEL driven by an electron beam with bell-shaped energy distribution as the following theorem.

Theorem 2.-There is a positive value of detuning, ${ }^{15}$

$$
\hat{\Delta}^{+}=p_{1}-\frac{P V \int_{-\infty}^{\infty} d \hat{P} \frac{d \hat{F}(\hat{P})}{d \hat{P}} \frac{1}{\hat{P}-p_{1}}}{1-\hat{\Lambda}_{p}^{2} P V \int_{-\infty}^{\infty} d \hat{P} \frac{d \hat{F}(\hat{P})}{d \hat{P}} \frac{1}{\hat{P}-p_{1}}},
$$

indicating the high-frequency cutoff of a 1D FEL amplification with bell-shaped electrons' energy distribution. For values of detuning below the threshold, the FEL has one growing mode, i.e., it always is unstable. Above the threshold none of the modes are growing.

Equation (50) combined with Eq. (2) gives us the maximal frequency amplified by a 1D FEL,

$$
\omega^{+} \equiv \frac{k_{w}+\Gamma \hat{\Delta}^{+}}{v_{z}^{-1}-c^{-1}}
$$

\footnotetext{
${ }^{14}$ This is not always true for energy distribution with multiple maxima.

${ }^{15}$ When $\hat{F}(\hat{P})$ depends solely on $\left(\hat{P}-p_{1}\right)^{2}$, typically, there are no singularities in the integrand, and hence no need to take the principal value of the integration.
} 
TABLE I. Cutoff frequency of FEL with bell-shaped energy distributions.

\begin{tabular}{lll}
\hline \hline Distribution function & Formula & $\hat{\Delta}^{*}$ \\
\hline Lorentzian & $\hat{F}(\hat{P})=\frac{1}{\sqrt{2} \pi \sigma\left(1+\hat{P}^{2} / 2 \sigma^{2}\right)}$ & $\frac{1}{2 \sigma^{2}+\hat{\Lambda}_{p}^{2}}$ \\
$\kappa-n$ & $\hat{F}(\hat{P})=\frac{\Gamma(n)}{\sqrt{2 \pi n} \Gamma(n-1 / 2)} \frac{1}{\sigma\left(1+\hat{P}^{2} / 2 n \sigma^{2}\right)^{n}}$ & $\frac{2 n-1}{2 n \sigma^{2}+(2 n-1) \hat{\Lambda}_{p}^{2}}$ \\
Gaussian & $\hat{F}(\hat{P})=\frac{1}{\sqrt{2 \pi} \sigma} e^{-\left(\hat{P}^{2} / 2 \sigma^{2}\right)}$ & $\frac{1}{\sigma^{2}+\hat{\Lambda}_{p}^{2}}$ \\
\hline \hline
\end{tabular}

with collinear propagation of the TEM wave and the electron beam. According to Eq. (6), in a 1D FEL where the TEM wave is traveling noncollinearly with the electron beam, this frequency can be generalized to

$$
\omega^{+}\left(k_{\perp}\right) \equiv \frac{k_{w}+\Gamma\left(\hat{\Delta}^{+}-\hat{k}_{\perp}^{2}\right)}{v_{z}^{-1}-c^{-1}} .
$$

Table I gives the expressions for the cutoff detuning parameters calculated from Eq. (50) for various frequently used energy distributions with the maximum located at
$\hat{P}=p_{1}=0$. The general dependence of high-frequency cutoff on the energy spread and space-charge parameter has the form $\hat{\Delta}^{*}=\left(a \sigma^{2}+\hat{\Lambda}_{p}^{2}\right)^{-1}$, with the coefficient $a$ determined by the specific form of the energy distribution.

As a specific example, we consider a Gaussian energy distribution:

$$
\hat{F}(\hat{P})=\frac{1}{\sqrt{2 \pi} \sigma} e^{-\left(\hat{P}^{2} / 2 \sigma^{2}\right)} .
$$

Inserting Eq. (53) into Eq. (14) yields

$$
w(i t)=i\left\{t-\frac{1-t \hat{\Lambda}_{p}^{2}}{\sigma^{2}}\left[1-\sqrt{\pi}\left(\frac{\hat{\Delta}-t}{\sqrt{2} \sigma}\right) \operatorname{Erfi}\left(\frac{\hat{\Delta}-t}{\sqrt{2} \sigma}\right) e^{\left[(\hat{\Delta}-t)^{2} / 2 \sigma^{2}\right]}\right]\right\}-\left.\left(1-t \hat{\Lambda}_{p}^{2}\right) \pi \frac{d \hat{F}(\hat{P})}{d \hat{P}}\right|_{\hat{P}=\hat{\Delta}-t},
$$

where we used the relation

$$
P V \int_{-\infty}^{\infty} \frac{x e^{-x^{2}}}{x-a} d x=\sqrt{\pi}-\pi a \operatorname{Erfi}(a) e^{-a^{2}} .
$$

Figure 4 shows the evolution of contour $\mathcal{D}_{1}$ with detuning, denoting that the winding number, and hence, the number of growing modes, changes from 1 to 0 as the detuning increases above 0.8. Figure 5 illustrates the numerical solution of the dispersion relation, Eq. (4), with the dispersion integral given by [2]

$$
\begin{aligned}
D(s)= & \frac{i}{\sigma^{2}}-\frac{i \sqrt{\pi / 2}}{\sigma^{3}} \\
& \times(s-i \hat{\Delta}) \exp \left[\frac{(s-i \hat{\Delta})^{2}}{2 \sigma^{2}}\right]\left[1-\operatorname{Erf}\left(\frac{s-i \hat{\Delta}}{\sqrt{2} \sigma}\right)\right] .
\end{aligned}
$$

There are full agreements between the numerical solutions and our theoretical conclusion on the number of growing modes and the cutoff frequency as shown in Table I.

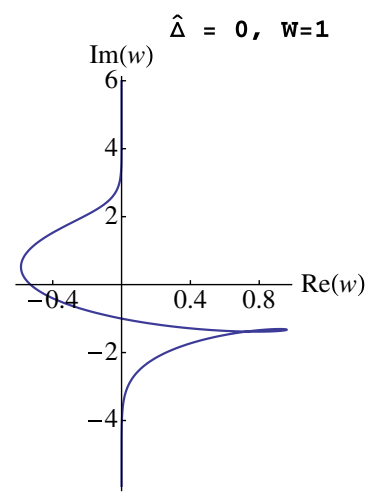

(a)

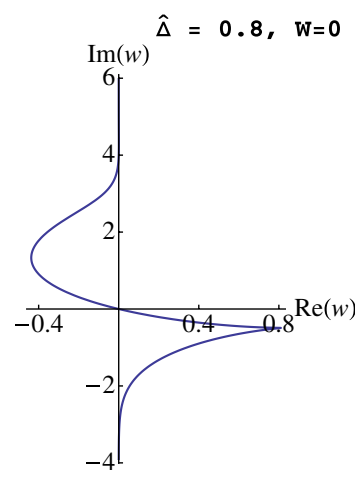

(b)

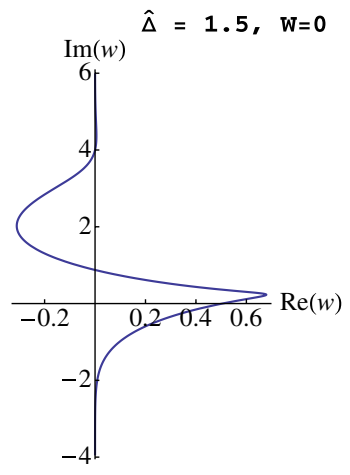

(c)

FIG. 4. Plots $w(i t)$ calculated from Eq. (54). The following parameters are used in these plots: $\hat{\Lambda}_{p}=0.5, \sigma=1$, and $\hat{\Delta}=0,0.8,1.5$ (from left to right). 


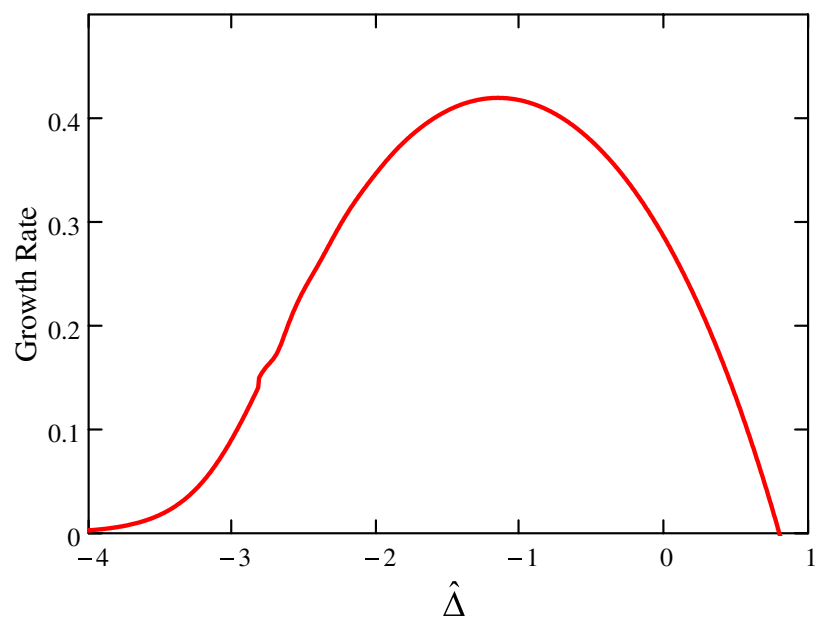

FIG. 5. Numerical solution of FEL growth rate for a Gaussian energy distribution. The growth rate was obtained by numerically solving Eq. (4) with $\hat{\Lambda}_{p}=0.5$ and $\sigma=1$.

\section{ENERGY DISTRIBUTION WITH MULTIPLE EXTREMA}

For an energy distribution having multiple extrema, the number of growing roots of DR strongly depends on the distribution's specific form. To proceed, we take an energy distribution of the following form:

$$
\begin{aligned}
\hat{F}(\hat{P})= & \frac{A}{\sqrt{2 \pi} \sigma_{1}} \exp \left[-\frac{\left(\hat{P}-\hat{P}_{1}\right)^{2}}{2 \sigma_{1}^{2}}\right]+\frac{1-A}{\sqrt{2 \pi} \sigma_{2}} \\
& \times \exp \left[-\frac{\left(\hat{P}-\hat{P}_{2}\right)^{2}}{2 \sigma_{2}^{2}}\right] .
\end{aligned}
$$

Inserting Eq. (57) into Eq. (14) gives

$$
\begin{aligned}
w(i t)= & i t-i\left(1-t \hat{\Lambda}_{p}^{2}\right)\left[A F_{1}^{I}(t)+(1-A) F_{2}^{I}(t)\right] \\
& -\pi\left(1-t \hat{\Lambda}_{p}^{2}\right)\left[A F_{1}^{R}(t)+(1-A) F_{2}^{R}(t)\right],
\end{aligned}
$$

with

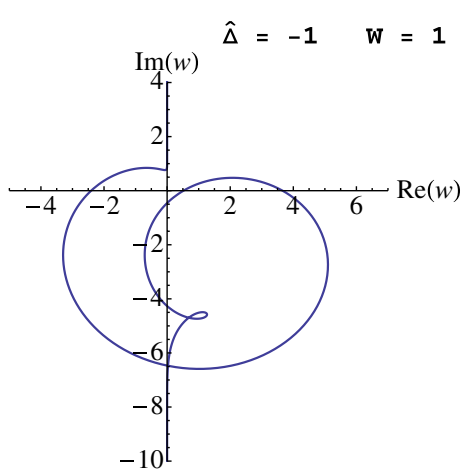

(a)

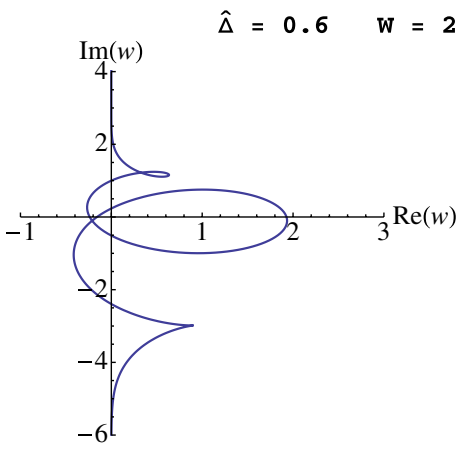

(b)

$$
F_{j}^{R}(t) \equiv-\frac{\hat{\Delta}-t-\hat{P}_{j}}{\sqrt{2 \pi} \sigma_{j}^{3}} \exp \left[-\frac{\left(\hat{\Delta}-t-\hat{P}_{j}\right)^{2}}{2 \sigma_{j}^{2}}\right] \text { for } j=1,2,
$$

and

$$
\begin{aligned}
F_{j}^{I}(t) \equiv & \frac{1}{\sigma_{j}^{2}}-\frac{\sqrt{\pi / 2}}{\sigma_{j}^{3}}\left(\hat{\Delta}-t-\hat{P}_{j}\right) \operatorname{Erfi}\left(\frac{\hat{\Delta}-t-\hat{P}_{j}}{\sqrt{2} \sigma_{j}}\right) \\
& \times \exp \left[-\frac{\left(\hat{\Delta}-t-\hat{P}_{j}\right)^{2}}{2 \sigma_{j}^{2}}\right] \text { for } j=1,2 .
\end{aligned}
$$

Figure 6 depicts the contour $\mathcal{D}_{1}$ with the energy distribution shown in Fig. 7(a), as calculated from Eq. (58), for three different detuning states. As determined from the winding number, the numbers of growth roots are $Z=1$ for $\hat{\Delta}=-1, Z=2$ for $\hat{\Delta}=0.6$, and $Z=0$ for $\hat{\Delta}=4$, agreeing with the numerical solutions of the dispersion relation, Eq. (4), as shown in Fig. 7(b). In obtaining that figure, we employed the following relation:

$$
\begin{aligned}
D(s)= & A\left\{\frac{i}{\sigma_{1}^{2}}-\frac{i \sqrt{\pi / 2}}{\sigma_{1}^{3}}\left(s-i \hat{\Delta}+i \hat{P}_{1}\right)\right. \\
& \left.\times \exp \left[\frac{\left(s-i \hat{\Delta}+i \hat{P}_{1}\right)^{2}}{2 \sigma_{1}^{2}}\right]\left[1-\operatorname{Erf}\left(\frac{s-i \hat{\Delta}+i \hat{P}_{1}}{\sqrt{2} \sigma_{1}}\right)\right]\right\} \\
& +(1-A)\left\{\frac{i}{\sigma_{2}^{2}}-\frac{i \sqrt{\pi / 2}}{\sigma_{2}^{3}}\left(s-i \hat{\Delta}+i \hat{P}_{2}\right)\right. \\
& \left.\times \exp \left[\frac{\left(s-i \hat{\Delta}+i \hat{P}_{2}\right)^{2}}{2 \sigma_{2}^{2}}\right]\left[1-\operatorname{Erf}\left(\frac{s-i \hat{\Delta}+i \hat{P}_{2}}{\sqrt{2} \sigma_{2}}\right)\right]\right\} .
\end{aligned}
$$

Next, we followed the steps developed in Sec. IV to assess the number of growing modes as a function of detuning. The extrema of the distribution in Fig. 7(a) are located at

$$
\hat{P}=p_{n}=\{0.0060,1.3708,2.4998\},
$$

and the corresponding $D_{n}$ are obtained from Eq. (25):

FIG. 6. Contour plots of $w(i t)$ as calculated from Eq. (58) for the energy distribution shown in Fig. 7(a). In all three, we used $\hat{\Lambda}_{p}=1$. (a) The winding number for $\hat{\Delta}=-1$ is $W=1$, and hence, there is one growing root. (b) The winding number for $\hat{\Delta}=0.6$ is $W=2$, and hence, there are two growing roots. (c) The winding number for $\hat{\Delta}=4$ is $W=0$, and accordingly, there are no growing roots. 


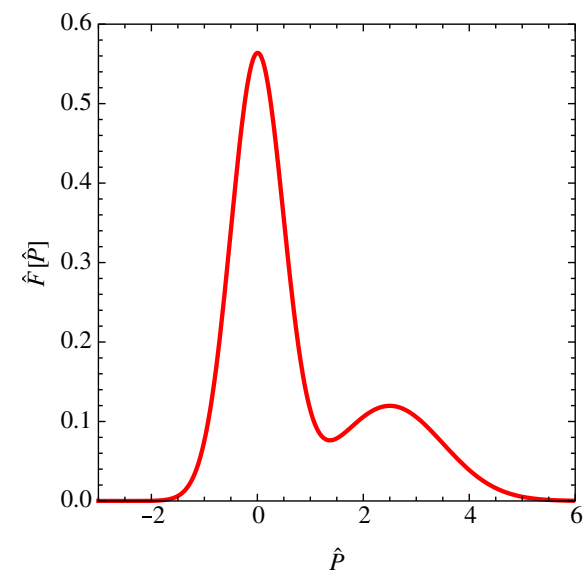

(a)

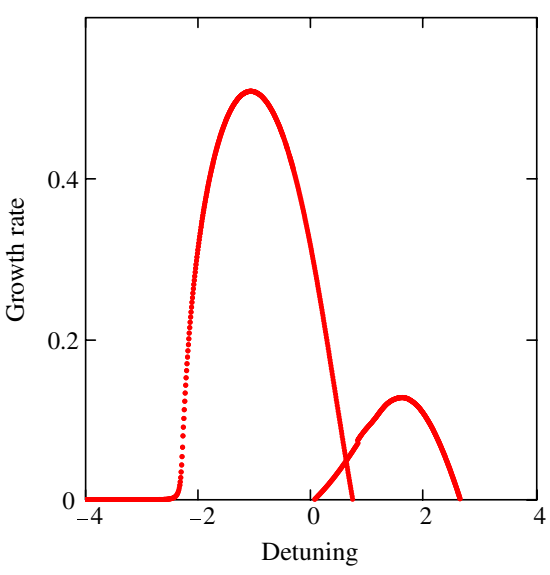

(b)

FIG. 7. Energy distribution with three local extrema and its corresponding FEL growth rate. (a) The energy distribution as described in Eq. (57) with $A=0.7, \sigma_{1}=0.5, \hat{P}_{1}=0, \sigma_{2}=1$, and $\hat{P}_{2}=2.5$. (b) The FEL growth rate for the energy distribution shown in (a) with $\hat{\Lambda}_{p}=1$.

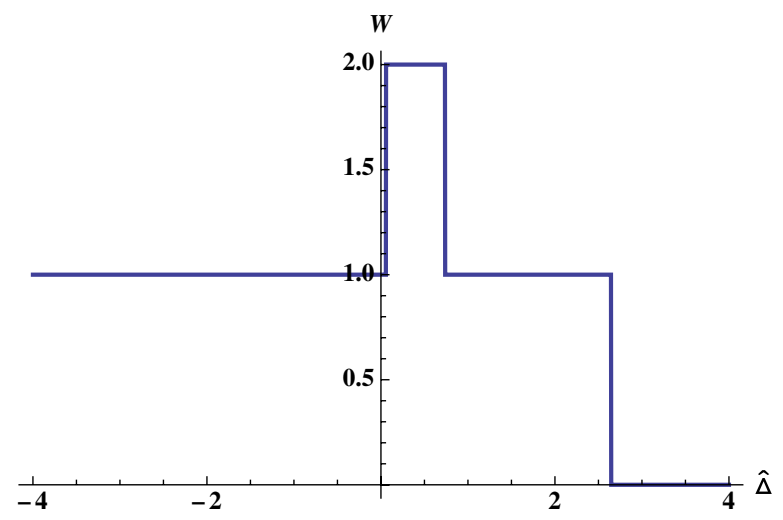

FIG. 8. The number of FEL growing modes calculated from Eq. (35) for an electron beam with the energy distribution shown in Fig. 7(a), and $\hat{\Lambda}_{p}=1$.

$$
D_{n}=\{2.7230,-0.5676,0.1705\} .
$$

Inserting $\hat{\Lambda}_{p}=1$ and Eq. (60) into Eq. (40) yields

$$
\hat{\Delta}_{n}^{+}=\{0.7374,0.0582,2.6455\} .
$$

Equation (61) shows the values of detuning where the number of growing mode changes. Applying Eq. (35) with $\hat{\Lambda}_{p}=1$ and $D_{n}$ given in Eq. (60), we obtained the number of growing modes as a function of detuning (Fig. 8); they fully agree with the direct numerical solutions of the dispersion relation illustrated in Fig. 7(b).

\section{SUMMARY}

In this paper, we show that if the energy distribution of the electron beam, $\hat{F}(\hat{P})$, is a smooth function and satisfies constraints listed in Eqs. (21) and (22), the maximal number of growing modes of the 1D FEL cannot exceed the number of maxima of the energy distribution. In addition, if $\hat{F}(\hat{P})$ has a bell-shaped form, there always is a single growing mode for frequencies (detuning) below a threshold, while there are none above the threshold. This threshold value is given by Eq. (50).

We developed steps for calculating the number of growing roots of the $1 \mathrm{D}$ dispersion relation for a general energy distribution. Our findings fully agree with the direct numerical solutions of the dispersion relation for all energy distributions considered here. For an electron energy distribution with more than one maximum, the high-frequency cutoff ceases to exist when the Penrose criterion is reached and the space-charge parameter is greater than certain value [7].

These results are directly applicable to an FEL with noncollinear propagation of radiation in a uniform electron beam.

\section{ACKNOWLEDGMENTS}

The authors would like to thank Michael Blaskiewicz for helpful discussions. This work was supported by Brookhaven Science Associates, LLC under Contract No. DE-AC02-98CH10886 with the U.S. Department of Energy.

\section{APPENDIX A: ON THE CONVERGENCE OF THE PRINCIPAL VALUE INTEGRAL}

Here we derive the condition wherein the principal value integral in Eq. (25) will be finite. We have an integral of the type

$$
I=P V \int_{-\infty}^{\infty} F^{\prime}(x) \frac{d x}{x-p} .
$$

Equation (A1) can be rewritten as 


$$
\begin{aligned}
I & =\int_{p}^{\infty} F^{\prime}(x) \frac{d x}{x-p}-\int_{p}^{-\infty} F^{\prime}(x) \frac{d x}{x-p} \\
& =\int_{0}^{\infty}\left[F^{\prime}(p+x)-F^{\prime}(p-x)\right] \frac{d x}{x} .
\end{aligned}
$$

Next, we divide the integration interval into $(0,1]$ and $(1, \infty)$ :

$$
I=I 1+I 2
$$

with

$$
I 1=\int_{0}^{1}\left[F^{\prime}(p+x)-F^{\prime}(p-x)\right] \frac{d x}{x},
$$

and

$$
I 2=\int_{1}^{\infty}\left[F^{\prime}(p+x)-F^{\prime}(p-x)\right] \frac{d x}{x} .
$$

Using the mean value theorem of integrals yields

$$
\begin{aligned}
|I 1| & \leq \int_{0}^{1}\left|F^{\prime}(p+x)-F^{\prime}(p-x)\right| \frac{d x}{x} \\
& =2 \int_{0}^{1}\left|F^{\prime \prime}\left(x^{*}\right)\right| d x \quad \text { for } x^{*} \in[p-x, p+x] .
\end{aligned}
$$

Assuming there exists a positive number $F_{\max }^{(2)}$ such that

$$
\left|F^{\prime \prime}(x)\right| \leq F_{\max }^{(2)} ; \quad \forall x \in[p-1, p+1],
$$

we obtain the upper limit of $I 1$ as

$$
|I 1| \leq 2 F_{\max }^{(2)}
$$

After integration by parts, Eq. (A5) becomes

$$
\begin{aligned}
I 2= & \int_{1}^{\infty}\left[F^{\prime}(p+x)-F^{\prime}(p-x)\right] \frac{d x}{x} \\
= & F(p-1)-F(p+1) \\
& +\int_{1}^{\infty}[F(p+x)-F(p-x)] \frac{d x}{x^{2}}
\end{aligned}
$$

Since $F(x) \geq 0, I 2$ has the following upper limit:

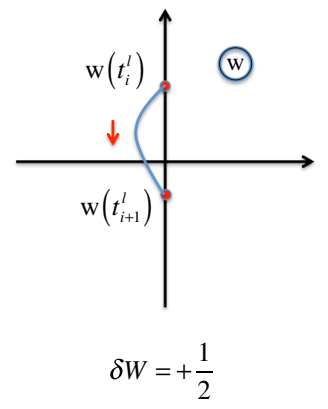

(a)

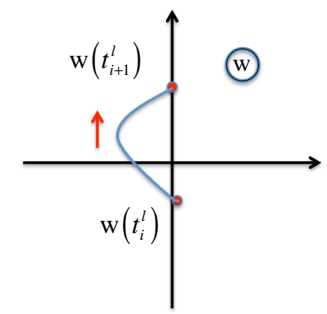

$\delta W=-\frac{1}{2}$

(b)

$$
\begin{aligned}
|I 2|= & \mid F(p-1)-F(p+1) \\
& +\int_{1}^{\infty}[F(p+x)-F(p-x)] \frac{d x}{x^{2}} \mid \\
\leq & F(p-1)+F(p+1) \\
& +\int_{-\infty}^{\infty}[F(p+x)+F(p-x)] d x \\
\leq & F(p-1)+F(p+1)+2 .
\end{aligned}
$$

Combining Eqs. (A8) and (A10) leads to

$$
\begin{aligned}
|I| & =|I 1+I 2| \leq|I 1|+|I 2| \\
& \leq 2 F_{\max }^{(2)}+F(p-1)+F(p+1)+2 .
\end{aligned}
$$

Hence, if $F(x)$ is finite and has finite second derivatives, the P.V. integral is finite.

\section{APPENDIX B: DERIVATION OF THE EQUATION TO CALCULATE WINDING NUMBER}

For each detuning segment in Eq. (33), we can define the sequence of intersections:

$$
t_{i}^{l}=\left\{t_{1}, \ldots, t_{l}, t_{0}, t_{l+1}, \ldots t_{2 M-1}\right\} \quad \text { for } i=1,2, \ldots, 2 M .
$$

Here, we introduce a new index, $i=1,2, \ldots, 2 M$, for the sequence to include $t_{0}$. In addition to the index $i$ of a pair of successive intersections, $\left\{t_{i}^{l}, t_{i+1}^{l}\right\}$, the signs of $\operatorname{Im} w\left(t_{i}^{l}\right)$ and $\operatorname{Im} w\left(t_{i+1}^{l}\right)$ determine the clockwise or counterclockwise winding of the contour segment starting from $t=t_{i}^{l}$ and ending at $t=t_{i+1}^{l}$. The contour segment with ending points $\left\{t_{i}^{l}, t_{i+1}^{l}\right\}$ does not affect winding number, provided that the signs of $\operatorname{Im} w\left(t_{i}^{l}\right)$ and $\operatorname{Im} w\left(t_{i+1}^{l}\right)$ are the same. On the contrary, if they are opposite, the contour segment changes the winding number by $\delta W= \pm 1 / 2$. Figure 9 illustrates the change in the winding number for odd and even $i$. The first and last segments, with ending points $\left\{\infty, t_{1}^{l}\right\}$ and $\left\{t_{2 M}^{l},-\infty\right\}$ respectively, may also contribute to the change in the winding numbers as illustrated in Fig. 10. The total

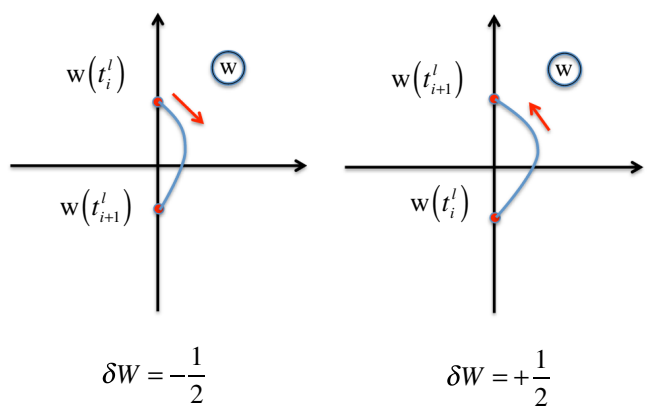

(c)

FIG. 9. The pairs of intersections that affect the winding number of the contour. Parts (a) and (b) are the odd pair, $i=2 j-1$. Parts (c) and (d) are the even pair, $i=2 j$. 


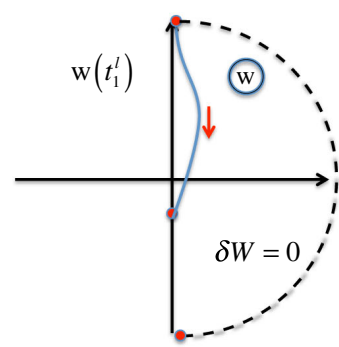

(a)

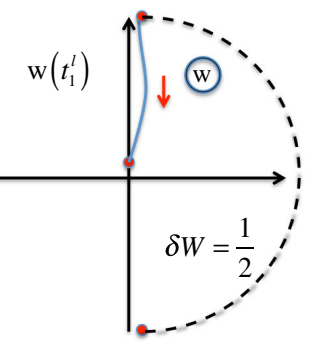

(b)

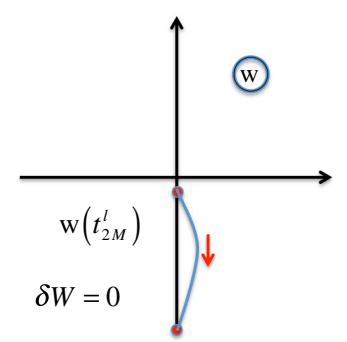

(c)

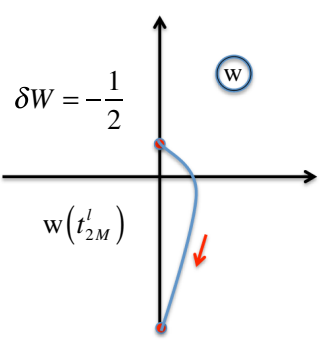

(d)

FIG. 10. The contribution from the first- and the last-intersecting points to the winding number of the contour. Parts (a) and (b) represent contributions from the first intersection. Parts (c) and (d) are contributions from the last intersection.

winding number of the full contour $\mathcal{D}$ is the sum of two parts; viz. from the $\mathcal{D}_{1}$ and the $\mathcal{D}_{2}$. The latter is included in Figs. 10(a) and 10(b). The overall contribution rule can be expressed as a simple formula:

$$
\begin{aligned}
\delta W_{0} & =\frac{1}{2} \theta\left[\operatorname{Im} w\left(t_{1}^{l}\right)\right] \\
\delta W_{i} & =\frac{(-1)^{i+1}}{2}\left\{\theta\left[\operatorname{Im} w\left(t_{i}^{l}\right)\right]-\theta\left[\operatorname{Im} w\left(t_{i+1}^{l}\right)\right]\right\} ; \\
i & =1,2 M-1 ; \quad \delta W_{2 M}=-\frac{1}{2} \theta\left[\operatorname{Im} w\left(t_{2 M}^{l}\right)\right] ;
\end{aligned}
$$

where $\delta W_{0}$ is contribution from the first and $\delta W_{2 M}$ is that from the last segment.

Summing up the three terms in Eq. (B2) gives

$$
W=\sum_{i=1}^{2 M}(-1)^{i+1} \theta\left[\operatorname{Im} w\left(t_{i}^{l}\right)\right]
$$

with its implicit dependence both on $l$ and $\hat{\Delta}$. Substituting $t_{i}^{l}=\left\{t_{1}, \ldots, t_{l}, t_{0}, t_{l+1}, \ldots t_{2 M-1}\right\}$ into Eq. (B3), we obtain a general equation for the winding number:

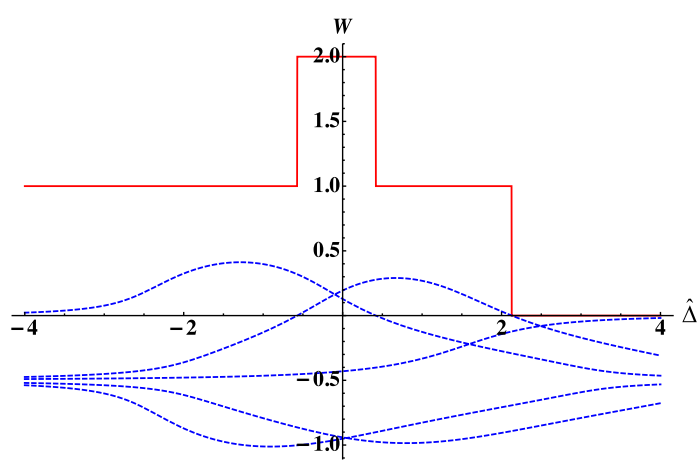

(a)

$$
\begin{aligned}
W(\hat{\Delta}) & =(-1)^{l(\hat{\Delta})}+\sum_{n=1}^{2 M-1}(-1)^{n+\theta[l(\hat{\Delta})-n]} \theta\left[\operatorname{Im} w\left(t_{n}\right)\right] \\
l(\hat{\Delta}) & =\sum_{n=1}^{2 M-1} \theta\left(\hat{\Delta}-\hat{\Lambda}_{p}^{-2}-p_{n}\right) ;
\end{aligned}
$$

wherein we took into account that $\theta\left[\operatorname{Im} w\left(t_{0}\right)\right]=$ $\theta\left(\hat{\Lambda}_{p}^{-2}\right)=1$. We can make the formulas for the winding number even more explicit using Eq. (26):

$$
W(\hat{\Delta})=(-1)^{l(\hat{\Delta})}+\sum_{n=1}^{2 M-1}(-1)^{n+\theta[l(\hat{\Delta})-n]} \theta\left(A_{n} \hat{\Delta}-B_{n}\right) .
$$

\section{APPENDIX C: EXAMPLE FOR BI-LORENTZIAN ENERGY DISTRIBUTION}

We present here an example of applying the method developed in Sec. III to calculate the number of growing roots for a bi-Lorentzian energy distribution, and to demonstrate the absence of a high-frequency cutoff for large space-charge. In previous sections, we show that for electron energy distribution having a single maximum, the

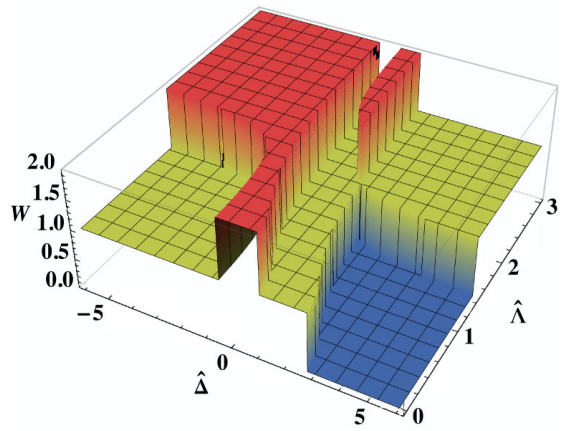

(b)

FIG. 11. The number of growing modes for an electron beam with bi-Lorentzian energy distribution as described in Eq. (C1). (a) The abscissa represents the normalized detuning and the ordinate is either the winding number (red solid curve) or the normalized growth rate (blue dashed curves). The winding number was calculated from Eq. (35) for $\zeta=\sqrt{3}, \hat{\Lambda}_{p}=0.5$, and $\sigma=0.5$. The blue curves are growth rate obtained by solving Eq. (4) with the dispersion integral given by Eqs. (C5) and (C6). (b) The number of growing modes as a function of the space-charge parameter and the normalized detuning as calculated from Eq. (35) for $\zeta=\sqrt{3}$ and $\sigma=0.5$. 
growing solution of the dispersion relation has a highfrequency cutoff. However, for a distribution with multiple extrema, the high-frequency cutoff may not exist. For simplicity, we consider the energy distribution of the following form:

$$
\hat{F}(\hat{P})=\frac{1}{2 \pi \sigma}\left[\frac{1}{1+\left(\frac{\hat{P}}{\sigma}-\zeta\right)^{2}}+\frac{1}{1+\left(\frac{\hat{P}}{\sigma}+\zeta\right)^{2}}\right] .
$$

The extrema of Eq. (C1) are located at

$$
\begin{gathered}
p_{1}=-\sigma \sqrt{2 \zeta \sqrt{1+\zeta^{2}}-1-\zeta^{2}}, \\
p_{2}=0,
\end{gathered}
$$

and

$$
p_{3}=\sigma \sqrt{2 \zeta \sqrt{1+\zeta^{2}}-1-\zeta^{2}}
$$

The dispersion function then can be evaluated at the extrema by inserting Eqs. (C2)-(C4) into Eq. (25) as

$$
D_{1}=D_{3}=\frac{1}{\sigma^{2}} \frac{1+\zeta^{2}-\sqrt{\zeta^{2}+\zeta^{4}}}{4\left(1+\zeta^{2}\right)\left(1+2 \zeta^{2}-2 \sqrt{\zeta^{2}+\zeta^{4}}\right)},
$$

and

$$
D_{2}=\frac{1}{\sigma^{2}} \frac{1-\zeta^{2}}{\left(1+\zeta^{2}\right)^{2}}
$$

Inserting Eqs. (C5) and (C6) into Eq. (40) and using Eq. (35), we obtain the number of growing roots of the dispersion relation as a function of detuning for $\zeta=\sqrt{3}$, $\hat{\Lambda}_{p}=0.5$, and $\sigma=0.5$ as shown in Fig. 11(a), that also shows the roots found by direct numerical solution, which fully agree with the calculations from Eq. (35).

In Fig. 11(b), the number of growing modes is plotted as a function of the space-charge parameter $\hat{\Lambda}_{p}$ and the normalized detuning $\hat{\Delta}$, showing that the high-frequency cutoff ceases to exist when the space-charge parameter is greater than certain value. The absence of the highfrequency cutoff is related closely to the two-stream instability of electron plasma when the Penrose instability criterion is reached.

[1] A. W. Chao and M. Tigner, Handbook of Accelerator Physics and Engineering (World Scientific Publishing Co. Pte. Ltd., New Jersey, 1998).

[2] E. L. Saldin, E. A. Schneidmiller, and M. V. Yurkov, The Physics of Free Electron Lasers (Springer, New York, 1999).

[3] S. Webb, V. N. Litvinenko, and G. Wang, Phys. Rev. ST Accel. Beams 15, 080701 (2012).

[4] S. Webb, G. Wang, and V. Litvinenko, Phys. Rev. ST Accel. Beams 14, 051003 (2011).

[5] H. Nyquist, Bell Syst. Tech. J. 11, 126 (1932) [http://media .johnwiley.com.au/product_data/excerpt/14/07803602/ 0780360214.pdf].

[6] L.P. Pitaevskii and E. M. Lifshitz, Physical Kinetics (Elsevier, Singapore, 2008), Vol. 10.

[7] O. Penrose, Phys. Fluids 3, 258 (1960). 